\title{
Transplante lamelar de córnea associado à tatuagem estromal para tratamento de leucoma: relato de caso
}

\author{
Corneal lamelar transplantation associated with stromal tattooing for leukoma \\ treatment: case report
}

\author{
André Berg'er ${ }^{1}$ \\ Márcia Ferrari Perez ${ }^{2}$ \\ Henrique Santiago Baltar Pazos ${ }^{3}$ \\ Simone Grigoletto De Biase ${ }^{4}$ \\ José Álvaro Pereira Gomes ${ }^{5}$
}

\section{RESUMO}

A ceratopigmentação, ou tatuagem do estroma corneano, é método utilizado para tratar cosmeticamente lesões corneanas como leucoma e coloboma traumático de íris. Pode-se realizar a aplicação do pigmento pela micropunção estromal ou associada à ceratectomia ou ceratoplastia lamelar. Demonstramos um caso de tatuagem estromal corneana associada a ceratoplastia lamelar em paciente com leucoma corneano extenso, com irregularidade de superfície, que não tolerava lentes de contato e sem prognóstico visual. Houve também correção do estrabismo divergente no mesmo tempo cirúrgico. Após um ano de seguimento, o pigmento mantém-se impregnado no estroma corneano, proporcionando bom aspecto estético. Em conclusão, a técnica da tatuagem associada ao transplante lamelar da córnea constitui bom método para tratamento cosmético do leucoma central com irregularidades da superfície corneana.

Descritores: Opacidade da córnea; Substância própria; Transplante de córnea; Tatuagem; Técnicas cosméticas; Lasers de excimer; Ceratectomia fotorrefrativa; Córnea/ cirurgia; Humano; Masculino; Adulto; Relatos de casos [Tipo de publicação]

\section{INTRODUÇÃO}

A ceratopigmentação, ou tatuagem do estroma corneano, é um método utilizado há séculos para tratar cosmeticamente lesões corneanas como leucoma e coloboma traumático de íris ${ }^{(1-4)}$. Pode ser realizada com pigmentos metálicos (ouro, prata ou platina) ou pigmentos "não-metálicos", como a tinta da Índia, da China, entre outros ${ }^{(1,3)}$. Seu uso não se popularizou devido ao progresso das técnicas de ceratoplastia e lentes de contato cosméticas ${ }^{(2)}$. Entretanto, em casos específicos como naqueles em que os pacientes apresentam cicatriz corneana excêntrica ou intolerância às lentes de contato, a tatuagem corneana ainda permanece uma boa indicação ${ }^{(1,3-7)}$.

Existem diferentes técnicas para realização da tatuagem corneana ${ }^{(4,7)}$. Pode-se realizar a aplicação do pigmento pela micropunção estromal ou associada à ceratetomia ou ceratoplastia lamelar. Neste relato de caso, demonstramos um caso de tatuagem estromal corneana associada a ceratoplastia lamelar em um olho sem prognóstico visual.

\section{RELATO DE CASO}

E.S., 45 anos, masculino, chegou ao ambulatório de córnea do Instituto Suel Abujamra com história de trauma ocular no olho direito há aproximadamente 20 anos e glaucoma desde então. Sua maior queixa consistia no 
aspecto estético. Negava dor, não tolerava lentes de contato cosméticas e não desejava a evisceração.

Ao exame oftalmológico apresentava acuidade visual no olho direito (OD): sem percepção luminosa (SPL) e no olho esquerdo (OE): 20/20. Em relação à motilidade ocular extrínsica: exotropia (XT) $35^{\Delta}$. À biomicroscopia, apresentava em OD: leucoma central com irregularidade superficial corneana e depósito de cálcio subepitelial (Figura 1 - A, B), afacia e sinéquias posteriores. A avaliação do fundo de olho era prejudicado em OD e normal em OE. A pressão intraocular (PIO) era aproximadamente $30 \mathrm{mmHg}$ OD e $18 \mathrm{mmHg}$ OE.

Indicou-se transplante de córnea lamelar com tatuagem e correção do estrabismo.

\section{Técnica cirúrgia}

Sob anestesia local e sedação, a correção do XT foi feita com retrocesso de reto lateral OD de $8 \mathrm{~mm}$ e ressecção do reto medial de $6 \mathrm{~mm}$ OD, deixando o paciente ortotrópico. Em seguida, foi realizado o transplante lamelar de córnea. Inicialmente houve a preparação da córnea doadora. Para realizar a preparação da córnea doadora de maneira homogênea, seguiu-se a técnica descrita por Ching-Li Cheng na qual foi usada uma base constituída por prótese esférica envolta em um campo cirúrgico estéril, presa em sua base por uma pinça de campo ${ }^{(9)}$. Suturou-se a esclera que circunda a córnea doadora nesse "molde", mantendo o endotélio corneano voltado para o campo, com 4 pontos separados de seda 4.0. O objetivo foi o de simular a tensão ocular normal e facilitar a manipulação do enxerto pelo cirurgião no momento da delaminação, feita num plano de aproximadamente três quartos da espessura corneana. Em seguida, colocou-se a córnea doadora com a face endotelial para cima sobre o suporte do "punch" para transplante e fez-se a trepanação de $11 \mathrm{~mm}$. Para preparar a córnea receptora, procedeu-se a incisão límbica de $2 / 3$ de profundidade e a dissecção lamelar com uso de espátula específica. Após remoção do terço anterior da córnea doadora, foi realizada a impregnação do estroma do paciente com pigmento (Mahatme - Eye bank \& Eye Hospital, Nagpur, Índia). Esta foi previamente esterilizada em autoclave, conforme orientação do fabricante, e depois foi retirada a quantidade desejada de pigmento e misturada com solução salina. A obtenção da coloração é conseguida dependendo da quantidade de pigmento por campo de tatuagem, deixando-se livre as porções em que a íris do paciente eram saudáveis e concentrando-se mais pigmento no centro pupilar. A dispersão do pigmento foi realizada com uma espátula de íris convencional, com a preocupação de se deixar o contorno regular e evitar o excesso nas bordas. O excesso de pigmento foi retirado com auxílio de esponja de merocel. Após a aplicação do pigmento, realizou-se a sutura da córnea doadora com 24 pontos simples de nylon 10.0. Ao final do procedimento, fez-se recobrimento com membrana amniótica com face epitelial para baixo ("patch").

O paciente foi tratado, no pós-operatório imediato, com acetazolamida $\left(\right.$ Diamox $\left.^{\circledR}\right) 750 \mathrm{mg} / \mathrm{dia}$, devido ao aumento da PIO, acetato de prednisolona $1 \%$ (Predfort $^{\oplus}$ ) de $2 / 2 \mathrm{~h}$ e moxifloxacino $\left(\right.$ Vigamox $\left.^{\circledR}\right)$ de $6 / 6$ h. Tão logo se observou a diminuição da PIO, houve retirada da acetazolamida, e com duas semanas, suspendeu-se o moxifloxacino. A prednisolona $1 \%$ foi retirada paulatinamente, em esquema de redução, até ser substituída por prednisolona $0,1 \%$ e depois foi suspensa.

Houve excelente aspecto cosmético final (Figura 2 - A, B, C, D), ocultando o leucoma e melhorando a regularidade da superfície corneana. A satisfação foi manifesta desde o primeiro dia de pós-operatório. Houve reepitelização da córnea doadora no acompanhamento, sem evidência de processo inflamatório. O pigmento mantém-se estável após mais de 1 ano de acompanhamento.

Apesar da melhora cosmética evidente da córnea, o paciente manteve hiperemia conjuntival crônica e olho doloroso, provavelmente relacionada à doença de base. No seguimento pós-operatório, melhorou com uso de corticoesteróide tópico e oral e lubrificantes tópicos.
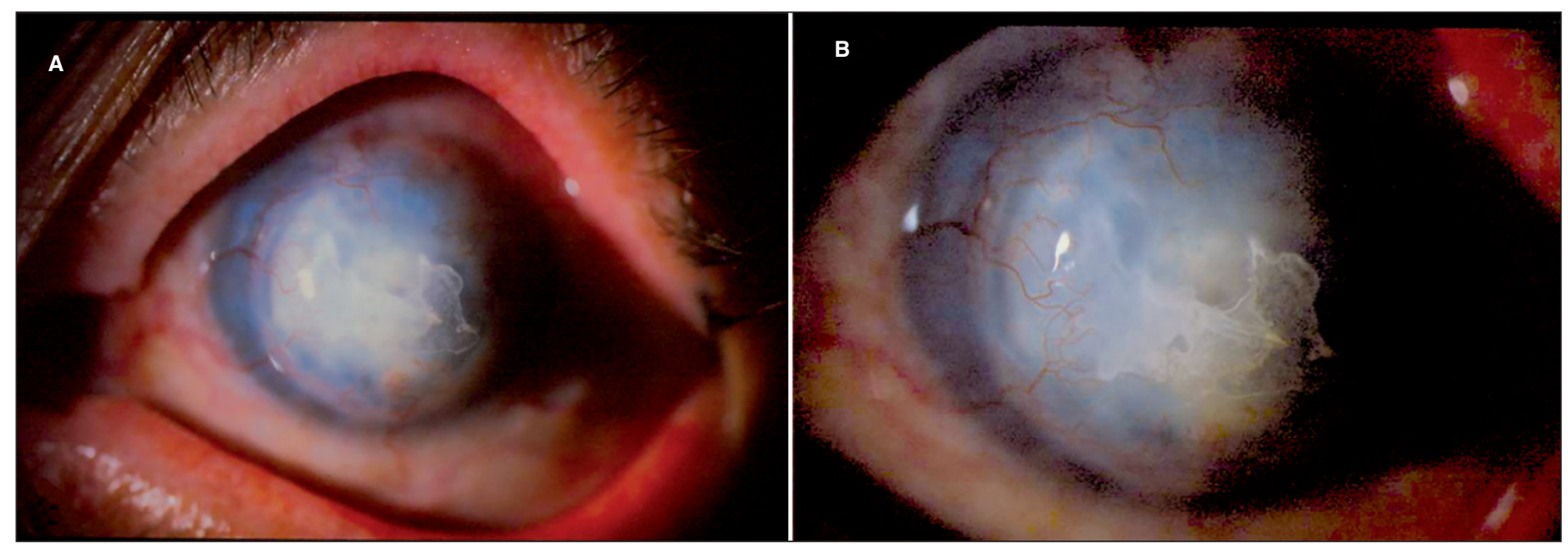

Figura 1 - A, B) Pré-operatório com leucoma, neovascularização e depósito de cálcio 

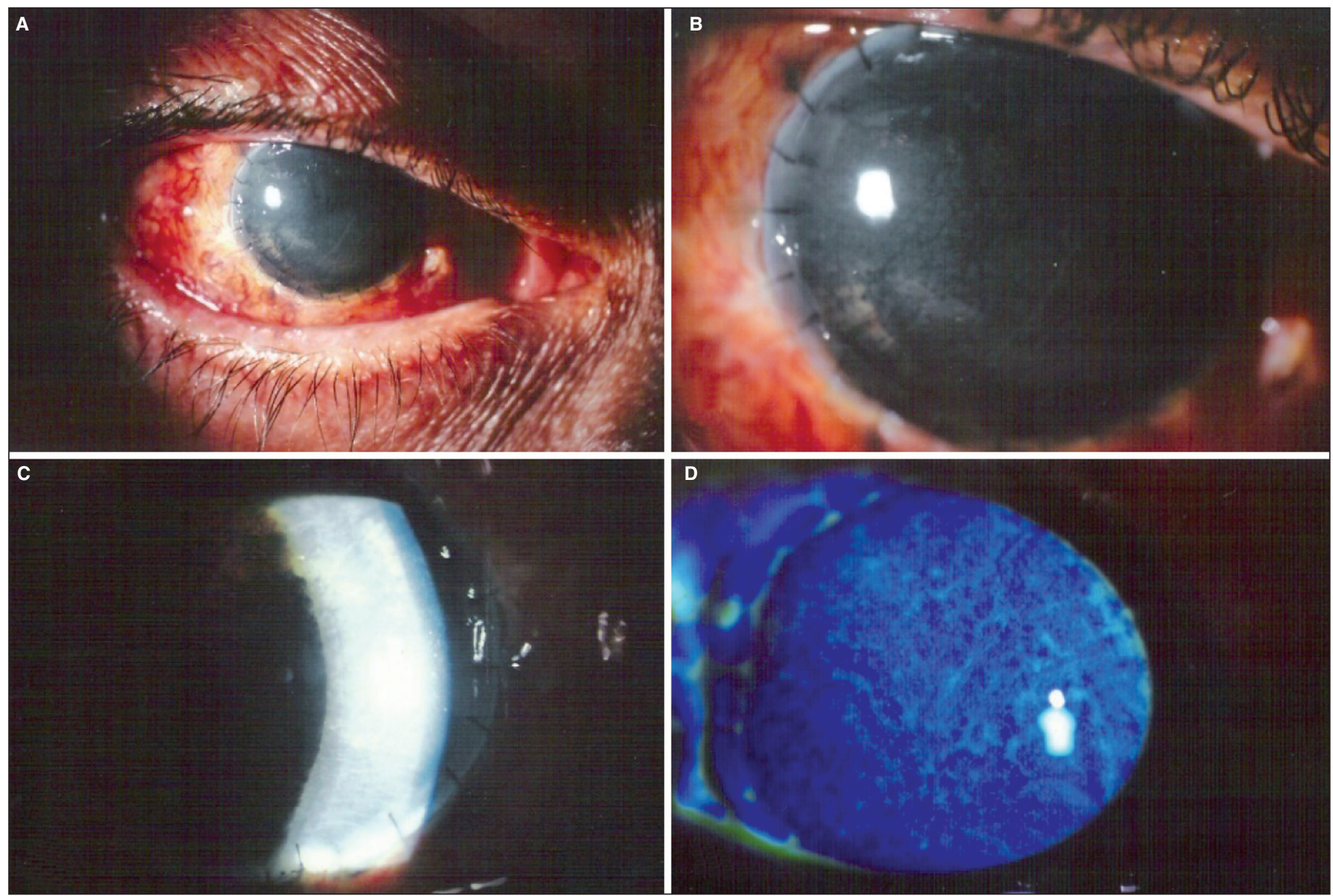

Figura 2 - A, B, C, D) Aspecto pós-operatório (2 meses) de transplante de córnea lamelar associado à tatuagem estromal. Note ótimo aspecto estético e completa re-epitelização da córnea doadora (D).

\section{DISCUSS ÃO}

A tatuagem, realizada de forma convencional, por meio de micropunturas estromais com pigmentos ${ }^{(1,3-7)}$ tem demonstrado eficácia em casos de leucomas pequenos e localizados. No entanto, não apresentam bom resultado em casos de leucoma total e irregularidade superficial da córnea, havendo desaparecimento progressivo do pigmento após poucas semanas do procedimento.

A técnica da tatuagem associada ao transplante lamelar, como apresentado neste caso, tem as vantagens de restituir a regularidade da superfície da córnea e manter por mais tempo o pigmento aderido nas camadas mais profundas da córnea. Essa observação foi por nós constatada pela manutenção do aspecto cosmético da córnea após 1 ano de seguimento ${ }^{(8)}$.

A segurança do transplante lamelar associado a tatuagem é relativamente alta, pelo que já foi publicado em relação aos pigmentos ${ }^{(2,4)}$ e por se tratar de transplante lamelar, realizado sem a abertura do globo ocular. A toxicidade dos pigmentos merece mais estudos ${ }^{(1)}$, mas se sabe que pigmentos não metálicos (ex: tinta da índia, como o que foi utilizado no caso descrito) são fagocitados pelos ceratócitos e aparentemente apresentam menor toxicidade e maior tempo de retenção na córnea do que pigmentos metálicos ${ }^{(2)}$. Não descartamos o quadro de hiperemia persistente ser decorrente de alguma manifestação tóxica do pigmento. Porém, baseado em outros estudos publicados ${ }^{(2,4)}$ e na experiência do cirurgião, isso parece ser pouco provável. Lembramos que o pigmento é patenteado por empresa indiana especificamente para uso ocular. Soma-se a esses fatos, o quadro anterior de glaucoma absoluto refratário e o histórico do trauma ocular, o que por si só explicariam o quadro de inflamação crônica. Além disso, o paciente apresenta rosácea, tendo melhorado com tratamento sistêmico com cloridrato de doxiciclina por via oral.

Em relação à técnica cirúrgica, o uso de trépanos de grandes dimensões teve por objetivo aproximar o tamanho do botão ao tamanho da córnea receptora, para dar a aparência estética de um olho normal, o que seria difícil conseguir com um trépano convencional, de aproximadamente $8 \mathrm{~mm}$ que não recobre áreas irianas periféricas. $\mathrm{O}$ intuito do uso de aparato para trepanação da córnea doadora, conforme descrição de Ching-Li Cheng foi o de auxiliar a dissecção lamelar anterior por cons- 
tituir suporte, servindo, em outras palavras, como uma câmara anterior artificial, em que não se necessita da viabilidade do endotélio ${ }^{(9)}$.

O uso da membrana amniótica como "patch" tem como objetivo auxiliar a epitelização da córnea transplantada ${ }^{(7)}$, conforme descrito por alguns autores, em casos de transplantes tectônicos da córnea. No caso relatado, a membrana amniótica começa a ser reabsorvida após 7 dias, após os quais pode-se observar completa epitelização da córnea transplantada.

A tatuagem estromal da córnea associada ao transplante lamelar parece ser um excelente método para correção cosmética de olhos sem visão, em pacientes que não toleram ou não desejam outro tipo de correção, proporcionando um resultado final aceitável e permitindo melhor convívio social. A disposição mais simétrica do pigmento, associado ao movimento natural que esta técnica proporciona, parece ser um dos principais motivos para o sucesso obtido. Mais casos com mais tempo de seguimento são necessários para se avaliar a estabilidade dos resultados e a toxicidade do pigmento.

\section{AGRADECIMENTOS}

Ao Dr. Renato Rezende Cordeiro.

\section{ABSTRACT}

Stromal cornea tattooing has been used for esthetic treatment of corneal scars as leukoma and iris coloboma. The coloring agent can be applied through stromal micropuncture or associated with lamellar keratectomy or keratoplasty. We report a case of stromal tattooing associated with lamellar keratectomy in a patient's eye that had extensive leukoma and irregular corneal surface. Visual acuity in the operated eye was no light percep- tion and the patient did not tolerate the use of cosmetic contact lens. Correction of divergent strabismus was also performed during the surgery. After one year follow-up, the ink is still impregnated in the corneal stroma, giving a good esthetic aspect. In conclusion, the technique of corneal tattooing associated with lamellar keratectomy is a good method for the cosmetic treatment of central leukoma with corneal irregularity.

Keywords: Corneal opacity; Corneal stroma; Corneal transplantation; Tattooing; Cosmetic techniques; Lasers, excimer, Photorefractive keratectomy; Cornea/surgery; Human; Male; Adult; Case reports [Publication type]

\section{REFERÊNCIAS}

1. Pitz S, Jahn R, Frisch L, Duis A, Pfeiffer N; Corneal tattooing: an alternative treatment for disfiguring corneal scars. Br J Ophthalmol. 2002;86(4): 397-9. Comment in: Br J Ophthalmol. 2002;86(12):1461.

2. Sekundo W, Seifert P, Seitz B, Loeffler KU. Long term ultrastructural changes in human corneas after tattooing with non-metallic substances. Br J Ophthalmol. 1999;83(2):219-24.

3. van der Velden EM, Samderubun EM, Kok JH. Dermatography as a modern treatment for coloring leucoma corneae. Cornea. 1994;13(4):349-53.

4. Mannis MJ, Eghbali K, Schwab IR. Keratopigmentation: a review of corneal tattooing. Cornea. 1999;18(6):633-7.

5. Panda A, Pangtey MS, Sony P. Corneal tattooing. Br J Ophthalmol. 2002; 86(12):1461. Comment in: Br Ophthalmol. 2002;86(4):397-9.

6. Lee JE, Jun JB, Choi HY, Oum BS, Lee JS. Corneal tattooing to mask subsequent opacification after amniotic membrane grafting for stromal corneal ulcer. Acta Ophthalmol Scand. 2006;84(5):696-8.

7. Pitz S, Jahn R, Frisch L, Duis A, Pfeiffer N. Corneal tattooing: an alternative treatment for disfiguring corneal scars. Br J Ophthalmol. 2002;86(4): 397-9. Comment in: Br J Ophthalmol. 2002;86(12):1461.

8. Panda A, Mohan M, Chawdhary S. Corneal tattooing-experiences with "lamellar pocket procedure". Indian J Ophthalmol [Internet]. 1984 [citado $2008 \mathrm{Mar}$ 27];32(5):408-11. Available from: http://www.ijo.in/text.asp?1984/32/5/408/ 27524.

9. Cheng CL, Theng JT, Tan DT. Compressive C-shaped lamellar keratoplasty a surgical alternative for the management of severe astigmatism from peripheral corneal degeneration. Ophthalmology. 2005;112(3):425-30. 